\title{
Quantum Decoherence of Two Qubits
}

\author{
Julius Helm and Walter T. Strunz \\ Institut für Theoretische Physik, Technische Universität Dresden, 01062 Dresden, Germany
}

(Dated: June 4, 2018)

\begin{abstract}
It is commonly stated that decoherence in open quantum systems is due to growing entanglement with an environment. In practice, however, surprisingly often decoherence may equally well be described by random unitary dynamics without invoking a quantum environment at all. For a single qubit, for instance, pure decoherence (or phase damping) is always of random unitary type. Here, we construct a simple example of true quantum decoherence of two qubits: we present a feasible phase damping channel of which we show that it cannot be understood in terms of random unitary dynamics. We give a very intuitive geometrical measure for the positive distance of our channel to the convex set of random unitary channels and find remarkable agreement with the so-called Birkhoff defect based on the norm of complete boundedness.
\end{abstract}

PACS numbers: 03.65.Yz,03.67.Pp,03.65.-w

\section{INTRODUCTION}

The loss of coherence in quantum systems is the hurdle that needs to be overcome in attempts to make use of quantum mechanics on larger and larger scales, most notably for quantum information tasks [1, 2]. Decoherence disentangles quantum states [3, 4, 5], which is why for experimental realizations of quantum information processors it is of fundamental importance to get a thorough understanding of the irreversible processes involved $[1,[3,6]$. Decoherence is also put forward to explain the appearance of classical properties in quantum systems [7, 8].

In open quantum system dynamics, damping (population transfer) is to be distinguished from decoherence (loss of phase relations in a certain basis). While damping necessarily implies decoherence, the converse need not be true for suitable interactions. Moreover, decoherence often occurs faster than damping, so that a description of the irreversible dynamics neglecting damping may be a valid approach for a short enough time. A prominent example is the quantum Brownian motion model [9], where the damping term is irrelevant during rapid decoherence [7, 8, 10]. Pure decoherence is usually referred to as dephasing or phase damping.

It is often stated that decoherence of an open quantum system is due to growing entanglement between system and environment [11, 12]. Nevertheless, more often than one might think, the resulting irreversible dynamics of the open system may be modeled entirely without invoking a quantum environment. Rather, the dynamics turns out to be indistinguishable from a random unitary (RU) evolution, which can be thought of as originating from classical fluctuations - sometimes called "random external fields" [1, 13] (see also the corresponding discussion in 7]). Note that in one of the most detailed experimental studies of decoherence in ion traps, classical fluctuations (i.e., RU dynamics) are used to cause controlled decoherence [14, 15]. In NMR decoherence studies, too, fluctuating classical fields are employed [3] .

There are many more relevant instances of decoher- ence that are of RU type. In fact, for a single qubit or qutrit, any possible phase damping is RU [16, 17]. Also, the very often employed model of Markovian dephasing (Lindblad master equation) [5, 18, 19] belongs to this class since any self-adjoint Lindblad operator may be identified with a white noise term in a suitable Hamiltonian. In this vein, the quantum Brownian motion master equation mentioned above (neglecting damping) follows from a white noise force term in the Hamiltonian, therefore being RU. Based on Feynman and Vernon's influence functional approach, one sees that the latter is not even restricted to the usual high temperature limit but can easily be extended to any temperature using colored noise - as long as times are short enough so that the nonMarkovian damping kernel may be neglected [20, 21].

We conclude that many widely used decoherence scenarios are of the RU class. Still, from the work of Landau and Streater [16] - which plays a central role for our results here - it is known that phase damping is not necessarily RU. However, there is no known simple criterion able to decide whether a given phase damping dynamics belongs to the RU class. From a more practical point of view, a test for a channel to be of non-RU type is also of relevance for quantum error correction [7] for it is known that such errors may not be fully corrected [22].

We deem it desirable to have a simple, explicit example of quantum decoherence at hand of which it is known that it cannot be expressed using stochastic Hamiltonians. Using a two qubit system we present a model of which we show that phase damping truly rests on growing entanglement with a quantum environment. Somewhat similar to studies by Havel and co-workers, our proposal may be implemented in NMR systems [3], and also in ion trap quantum computers [6].

In a first step, we choose as environment a single, third qubit. The proof that the corresponding decoherence cannot be understood in terms of RU dynamics follows immediately from the work of Landau and Streater (Sec. II). Remarkably, using the Bloch sphere picture, we find a nice geometrical measure (a volume) that indicates how "non-RU" the dynamics is. This quantity correlates sur- 
prisingly well with the distance of the quantum decoherence channel from the convex set of RU dynamics (using the so-called cb-norm, Sec. III). In Sec. IV we extend our model to include genuine irreversibility.

\section{PHASE DAMPING CHANNELS AND EXTREMALITY}

The dynamics of a quantum state $\varrho \rightarrow \varrho^{\prime}=\mathcal{E}[\varrho]=$ $\sum_{i} K_{i} \varrho K_{i}^{\dagger}$ is a completely positive map (or quantum channel) with Kraus operators $K_{i}$ [1] (neglecting initial correlations). A decoherence or phase damping channel belongs to the class of doubly stochastic channels. These are trace preserving and unital (mapping the identity onto itself), corresponding to $\sum_{i} K_{i}^{\dagger} K_{i}=\mathbb{1}$ and $\sum_{i} K_{i} K_{i}^{\dagger}=\mathbb{1}$, respectively. The question about the nature of the irreversibility (entanglement vs RU) is then in close analogy to the classical Birkhoff theorem, stating that every real doubly stochastic matrix can be written as a convex sum of permutations [16]. In the quantum case, this corresponds to the question of whether the set of doubly stochastic quantum channels is identical to the set of RU channels $\mathcal{E}[\varrho]=\sum_{i} p_{i} U_{i} \varrho U_{i}^{\dagger}$ with unitary $U_{i}$, $p_{i}>0$, and $\sum_{i} p_{i}=1$ (see [17, 23] for recent work on RU channels).

Decoherence or phase damping channels are among the simplest conceivable maps. They are defined by the requirement that, in the given basis $\{|n\rangle\}$ with $1 \leq n \leq d$, no population transfer takes place: $\langle n|\varrho| n\rangle=$ const. The only effect of the "environment" is thus to change coherences $\langle m|\varrho| n\rangle$ with $m \neq n$. Thus, the Kraus operators have to be diagonal in this basis, $K_{i}=\operatorname{diag}\left(a_{i 1}, a_{i 2}, \ldots, a_{i d}\right)$ and, correspondingly, the whole map $\varrho^{\prime}=\mathcal{E}[\varrho]$ is diagonal,

$$
\varrho_{m n}^{\prime}=\left\langle a_{n} \mid a_{m}\right\rangle \varrho_{m n}
$$

with $\left\{\left|a_{n}\right\rangle=\left(a_{1 n}, a_{2 n}, \ldots, a_{r n}\right)\right\}$ any set of $d$ normalized complex vectors [24, 25]. Phase damping channels are just the diagonal, doubly stochastic quantum channels.

If the phase damping channel $\mathcal{E}$ results from the coupling between the system and a quantum mechanical environment, the vectors $\left|a_{n}\right\rangle$ may be understood as relative quantum states of the environment, relative to the states of the distinguished basis [26]. Yet, this need not be: for a single qubit the most general phase damping channel

$$
\varrho^{\prime}=e^{-i \phi_{0} \sigma_{z}}\left(p \varrho+(1-p) \sigma_{z} \varrho \sigma_{z}\right) e^{i \phi_{0} \sigma_{z}}
$$

may obviously be obtained from $U_{\phi}=e^{-i \phi \sigma_{z}}$ with a random variable $\phi$ with $\phi_{0}=\langle\langle\phi\rangle\rangle$ and $p=\left\langle\left\langle\cos ^{2}\left(\phi-\phi_{0}\right)\right\rangle\right.$. Landau and Streater show in [16] that for the case of $d \geq 4$, e.g., for at least a two-qubit system, there exist non-unitary extremal maps in the set of diagonal doubly stochastic quantum channels: there are phase damping channels of two qubits that are not of RU type.

We set out to construct such a channel, which requires having a test for a channel's extremality. A given di-

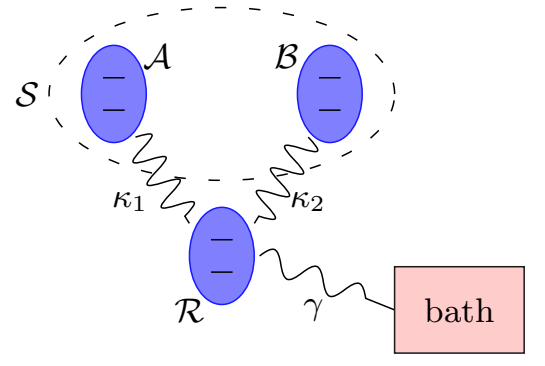

FIG. 1: (Color online) Phase damping channel on the system $\mathcal{S}$ of qubits $\mathcal{A}$ and $\mathcal{B}$. The "environment" initially consists of just a single qubit $\mathcal{R}$, which is later coupled to an additional zero temperature bath.

agonal doubly stochastic channel $\mathcal{E}$ is extremal iff it admits a Kraus representation $\mathcal{E}[\varrho]=\sum_{i=1}^{r} K_{i} \varrho K_{i}^{\dagger}$, where $\left\{K_{i}^{\dagger} K_{j}\right\}_{i, j=1}^{r}$ is a linear independent set of matrices [16]. This linear independence is equivalent to a quality of the associated vectors $\left\{\left|a_{1}\right\rangle, \ldots,\left|a_{d}\right\rangle\right\} \subset \mathbb{C}^{r}$ called "full set of vectors" (FSOV) [16], which is attained if, for a complex matrix $M \in \mathbb{C}^{r \times r},\left\langle a_{n}|M| a_{n}\right\rangle=0 \forall n$ implies $M=0$. Note that in case of a two-qubit channel extremality implies $r \leq 2$ : for $r=1$ this is just unitary dynamics, so that $r=2$ gives the only possibility of an extremal, nonunitary phase damping channel, implying the $\left|a_{n}\right\rangle$ to be single-qubit states.

Based on this relation we give a simple test for extremality. With $\vec{\Sigma}:=(\mathbb{1}, \vec{\sigma})=\left(\mathbb{1}, \sigma_{x}, \sigma_{y}, \sigma_{z}\right)$ we denote the vector containing the usual basis of linear operators in two-dimensional Hilbert space. Then, for the states $\left|a_{n}\right\rangle$ of the environmental qubit, the Bloch representation reads $\left|a_{n}\right\rangle\left\langle a_{n}\right|=\frac{1}{2}\left(\mathbb{1}+\vec{b}_{n} \cdot \vec{\sigma}\right)=: \vec{B}_{n} \cdot \vec{\Sigma}$, where $\vec{B}_{n}=\frac{1}{2}\left(1, \vec{b}_{n}\right)$. When also rewriting $M$ in this basis, $M=\vec{K} \cdot \vec{\Sigma}$, with $\vec{K} \in \mathbb{C}^{4}$, the FSOV condition demands that $\left\langle a_{n}|M| a_{n}\right\rangle=\vec{B}_{n} \cdot \vec{K}=0$ for all $n$ implies $M=0$ and, accordingly, $\vec{K}=0$. Hence, the vectors $\left\{\vec{B}_{1}, \ldots, \vec{B}_{4}\right\}$ have to be linear independent, so that we get the following equivalence:

$$
\begin{gathered}
\left\{\left|a_{1}\right\rangle, \ldots,\left|a_{4}\right\rangle\right\} \\
\text { is a FSOV }
\end{gathered} \Leftrightarrow V_{t}:=\frac{1}{6} \operatorname{det}\left(\begin{array}{ccc}
1 & \cdots & 1 \\
\vec{b}_{1} & \cdots & \vec{b}_{4}
\end{array}\right) \neq 0 .
$$

In addition we arrive at a geometrical interpretation of the FSOV condition: the channel is extremal iff the Bloch vectors $\vec{b}_{n}$ do not point to the same hyperplane in $\mathbb{R}^{3}$, or, equivalently, iff the volume $V_{t}$ of the 3 -dimensional tetrahedron spanned by the Bloch vectors is different from zero (see also fig. 2 (c) ). Note that we here discuss the case of a system of two qubits only, the results can however be extended easily to arbitrary dimension. 


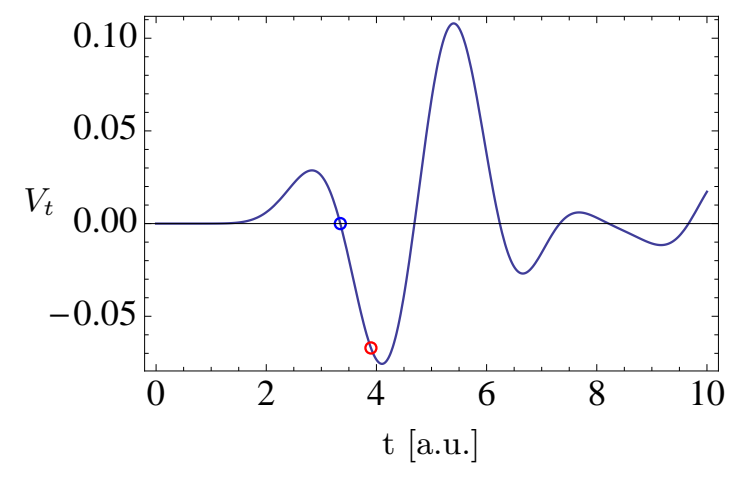

(a)

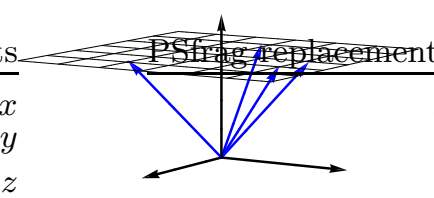

(b)

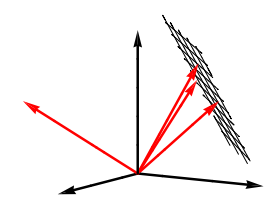

(c)
FIG. 2: (Color online) (a) The volume $V_{t}$ (3) against time $t$ for a given set of parameters $\kappa_{1}, \kappa_{2}, \vec{\Gamma}$. For values of $V_{t}=0$ [left circle and (b), exemplary] the corresponding Bloch vectors are coplanar, and the phase damping channel is RU. For $V_{t} \neq 0$ [right circle and (c), exemplary] the dynamical vectors $\left\{\left|\psi_{t}^{(n)}\right\rangle\right\}$ form a FSOV, so that the corresponding Bloch vectors are not coplanar. In this case, the model gives a quantum decoherence channel.

\section{QUANTUM DECOHERENCE DUE TO SINGLE-QUBIT "ENVIRONMENT"}

After having presented the technical prerequisites we now want to consider a simple model based on a twoqubit system $\mathcal{S}$ (qubits $\mathcal{A}$ and $\mathcal{B}$ ) interacting with a quantum mechanical "environment," consisting of only one single qubit $\mathcal{R}$ (cf. Fig. 11). Evolution of the compound system shall be described by the Hamiltonian

$$
H=H_{\mathcal{S}}+H_{\mathcal{I}}+H_{\mathcal{R}}
$$

where $H_{\mathcal{S}}=\Omega_{1} \sigma_{z}^{(\mathcal{A})}+\Omega_{2} \sigma_{z}^{(\mathcal{B})}$ denotes the system Hamiltonian, $H_{\mathcal{I}}=\kappa_{1} \sigma_{z}^{(\mathcal{A})} \sigma_{z}^{(\mathcal{R})}+\kappa_{2} \sigma_{z}^{(\mathcal{B})} \sigma_{z}^{(\mathcal{R})}$ describes the interaction between system and environment, and $H_{\mathcal{R}}=$ $\vec{\Gamma} \cdot \vec{\sigma}^{(\mathcal{R})}\left(\vec{\Gamma}=\left(\Gamma_{x}, \Gamma_{y}, \Gamma_{z}\right)\right)$ gives the free evolution of the reservoir qubit.

With the usual product initial state $\varrho \otimes \sigma$, for any given time $t$ the reduced dynamics of the two qubits defines a phase damping channel

$$
\mathcal{E}_{t}[\varrho]=: \varrho^{\prime}=\operatorname{tr}_{\mathcal{R}}\left(e^{-i H t}(\varrho \otimes \sigma) e^{i H t}\right) .
$$

Rewriting $H$ in the basis of the system's energy eigenstates, $H=\sum_{n=1}^{4}|n\rangle\langle n| \otimes \tilde{H}_{\mathcal{R}}^{(n)}$, and taking the initial state of the reservoir to be pure, i.e., $\sigma=\left|\psi_{0}\right\rangle\left\langle\psi_{0}\right|$, we get

$\varrho_{m n}^{\prime}=\operatorname{tr}\left(e^{-i \tilde{H}_{\mathcal{R}}^{(m)} t}\left|\psi_{0}\right\rangle\left\langle\psi_{0}\right| e^{i \tilde{H}_{\mathcal{R}}^{(n)} t}\right) \varrho_{m n}=\left\langle\psi_{t}^{(n)} \mid \psi_{t}^{(m)}\right\rangle \varrho_{m n}$, with the relative states of the environment $\left|\psi_{t}^{(n)}\right\rangle:=$ $e^{-i \tilde{H}_{\mathcal{R}}^{(n)} t}\left|\psi_{0}\right\rangle, n=1, \ldots, 4$. The extremality of the phase damping channel may now be checked by calculating the volume $V_{t}$ (3). We find that extremality requires the parameters of our model to meet essentially three conditions:

(I) Asymmetric coupling: $0 \neq \kappa_{1} \neq \kappa_{2} \neq 0$,

(II) $\Gamma_{x} \neq 0$ or $\Gamma_{y} \neq 0$, and

(III) $\Gamma_{z} \neq 0$.

For $V_{t}=0$ the channel is not only non-extremal, we can further show that it is also random unitary. First note that for a channel with $\vec{b}_{1}, \ldots, \vec{b}_{4}$ pointing to a plane parallel to the $x$-y-plane random unitarity follows immediately, for we can write $\left|\psi_{t}^{(n)}\right\rangle=\left(\sqrt{1-p} e^{i \varphi_{1}^{(n)}}, \sqrt{p} e^{i \varphi_{2}^{(n)}}\right)$ with the same $p$ for all $n=1, \ldots, 4$, resulting in the Kraus form $\mathcal{E}[\varrho]=(1-p) U_{1} \varrho U_{1}^{\dagger}+p U_{2} \varrho U_{2}^{\dagger}$. For arbitrary coplanar Bloch vectors $\vec{b}_{1}, \ldots, \vec{b}_{4}$ a suitable rotation of both the initial state $\left|\psi_{0}\right\rangle$ and the Hamiltonians $\tilde{H}_{\mathcal{R}}^{(n)}$ leaves the phase damping channel unaltered, whereas the plane spanned by the new Bloch vectors is again parallel to the $x$-y-plane.

In Fig. 2 (a) the volume $V_{t}$ is plotted as a function of time $t$ for a realization with conditions (I) - (III) met. Based on our considerations we can conclude that for almost all times the corresponding phase damping channel $\mathcal{E}_{t}$ is an extremal channel $\left(V_{t} \neq 0\right)$. From the decrease in Purity $P\left(\varrho^{\prime}\right)=\operatorname{tr}\left(\varrho^{\prime 2}\right)$, we can exclude unitary dynamics, assuring the channel to be a genuine quantum decoherence channel [cf. Fig. 4 (a), solid line].

We now want to quantify the "quantumness" of the decoherence by determining the norm distance of the obtained channel to the set of RU channels, also called the Birkhoff defect, $d_{B}$ [27]. The norm distance is calculated in terms of the cb-norm (norm of complete boundedness, for definitions and properties see [25, 28]). It involves numerical minimization over (a) the convex set of RU channels, and (b) equivalent operator sum representations of the channel occurring from the difference of the given phase damping channel and the corresponding random unitary channel. In order to find the global minimum we use several starting points, from where we alternately minimize with respect to (a) and (b). For the calculation of the cb-norm a slightly modified version of the algorithm described in [28] is used.

The Birkhoff defect shows a remarkable qualitative agreement with the absolute volume of the tetrahedron spanned by the four Bloch vectors $\left\{\vec{b}_{1}, \ldots, \vec{b}_{4}\right\}$ (see Fig. 3). Obviously, the tetrahedron volume not only enables to distinguish the different classes of dynamics, it also gives a quantitative measure of the quantumness of the channel. 


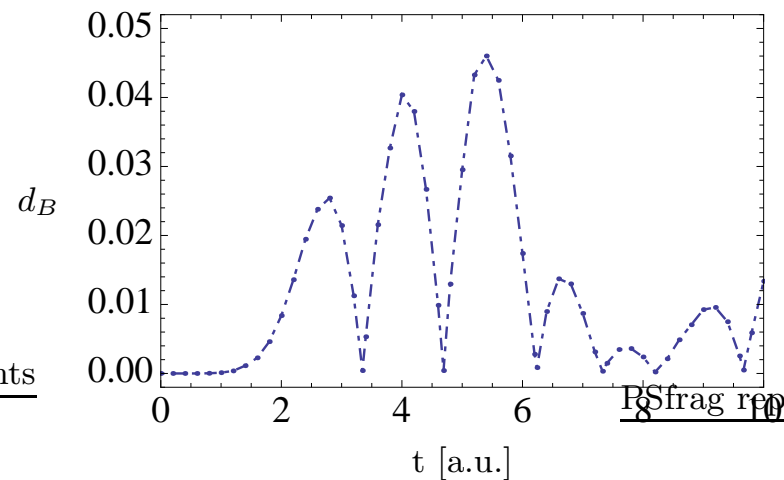

(a)

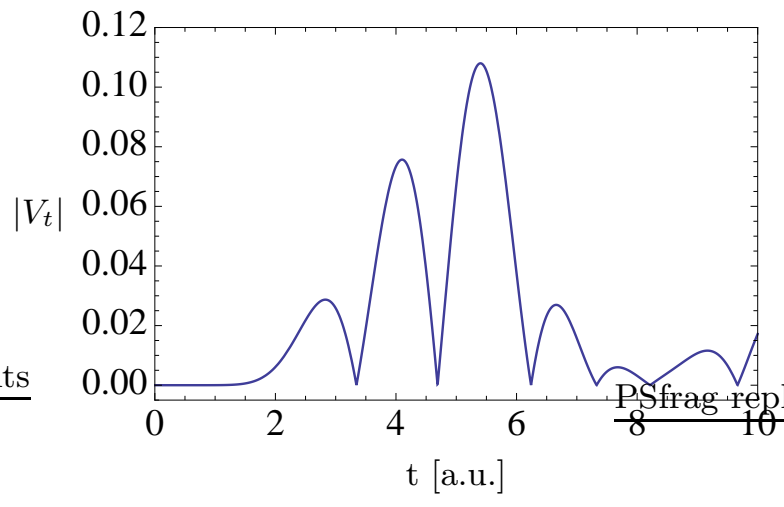

(b)

FIG. 3: (Color online) (a) the calculated cb-norm distance $d_{B}$ of our quantum phase damping channel to the set of random unitary channels. The qualitative agreement with (b) the absolute value $\left|V_{t}\right|$ of the volume spanned by the Bloch vectors characterizing the channel is remarkable.

\section{IRREVERSIBLE QUANTUM DECOHERENCE}

Clearly, the three-qubit model is fully reversible. In order to introduce irreversibility we include an additional damping of the reservoir qubit via spontaneous decay (cf. Fig. 10, leading to the Markovian master equation for the full density operator $\varrho_{\text {tot }}[29]$

$\dot{\varrho}_{\mathrm{tot}}=-i\left[H, \varrho_{\mathrm{tot}}\right]+\frac{\gamma}{2}\left(2 \sigma_{-} \varrho_{\mathrm{tot}} \sigma_{+}-\sigma_{+} \sigma_{-} \varrho_{\mathrm{tot}}-\varrho_{\mathrm{tot}} \sigma_{+} \sigma_{-}\right)$,

where $H$ is the Hamiltonian describing the original model (44), $\sigma_{ \pm}$are the raising and lowering operators acting on the reservoir qubit. The channel is again given through the reduced dynamics $\mathcal{E}_{t}[\varrho]=\operatorname{tr}_{\mathcal{R}}\left(\varrho_{\text {tot }}(t)\right)=: \varrho^{\prime}$. The influence of the additional damping may be seen in the

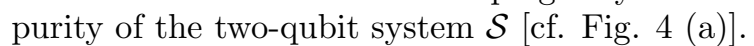

For this extended model the FSOV criterion is no longer suitable (the environment can obviously no longer be described as a single qubit). Yet, the Birkhoff defect $d_{B}$ gives a way of examining the nature of the channel. We observe [cf. Fig. 4 (b)] that for increasing coupling $\gamma$ of the environment qubit to the zero temperature bath the Birkhoff defect of an average channel decreases until,

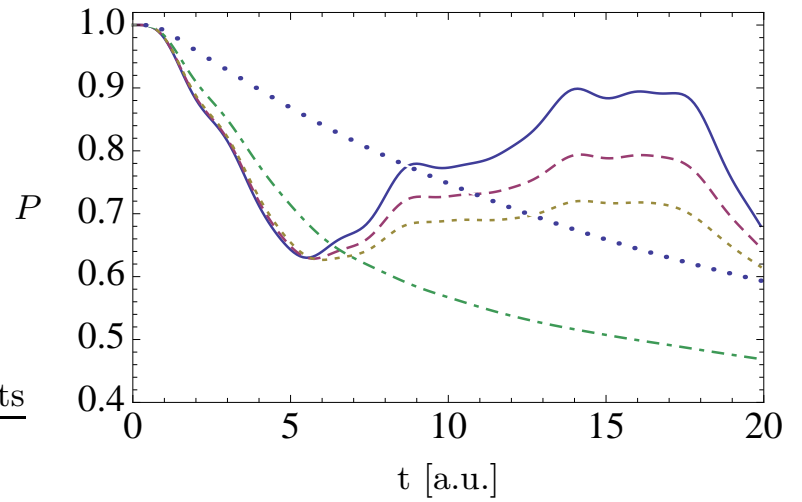

(a)

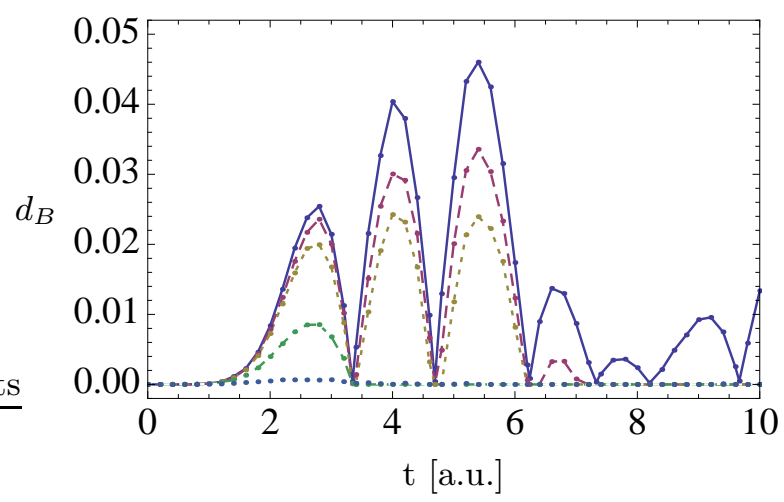

(b)

FIG. 4: (Color online) (a) Purity $P$ of the two-qubit system $\mathcal{S}$ and (b) Birkhoff defect $d_{B}$ of the quantum channel against time $\mathrm{t}$ for the single-qubit environment (solid line), and with additional coupling to a zero temperature bath with coupling strength $\gamma=0.05$ (long dashes), 0.1 (short dashes), 0.5 (dotdashed), and 2 (dotted).

for $\gamma$ large enough, it is zero for almost all times. Note, however, that for small coupling $d_{B}$ stays well above zero: we can still observe quantum decoherence.

\section{CONCLUSIONS}

To summarize, based on a simple model we are able to give a generic example of a feasible two-qubit decoherence channel that does not belong to the class of random unitary channels. Remarkably, we see a strong correlation between the Birkhoff defect and the volume of the tetrahedron spanned by the Bloch vectors of the relative states of the environment qubit. For an extension of our model including irreversibility, we see genuine quantum decoherence as long as the coupling to the bath is small enough. We hope that our model will help to further explore the difference between "classical" random unitary phase damping and "true" quantum decoherence and thus help to elucidate the true role of entanglement in open quantum system decoherence. 


\section{Acknowledgments}

The authors would like to thank Gernot Alber, Lajos Diósi, Hartmut Häffner, Florian Mintert, Carlos Pineda,
Stephan Rietzler, Thomas Seligman, Markus Tiersch, and Michael M. Wolf for useful discussions and hints. J.H. acknowledges support from the International Max Planck Research School Dresden.
[1] M.A. Nielsen and I.L. Chuang, Quantum Computation and Quantum Information (Cambridge University Press, Cambridge, U.K., 2007).

[2] N. Gisin et al., Rev. Mod. Phys. 74, 145 - 195 (2002).

[3] G. Teklemariam et al., Phys. Rev. A 67, 062316 (2003).

[4] T. Yu and J.H. Eberly, Phys. Rev. B 68, 165322 (2003).

[5] F. Mintert et al., Phys. Rep. 415, 207 - 259 (2005).

[6] H. Häffner et al., Phys. Rep. 469, 155 - 203 (2008).

[7] W.H. Zurek, Rev. Mod. Phys. 75, 715 - 775 (2003).

[8] E. Joos et al., Decoherence and the Appearance of a Classical World in Quantum Theory, 2nd ed. (Springer, New York, 2003).

[9] A. O. Caldeira and A. J. Leggett, Physica A 121, 587 (1983).

[10] D. Braun, F. Haake, and W.T. Strunz, Phys. Rev. Lett 86, 2913 (2001).

[11] R. Blume-Kohout and W.H. Zurek, Phys. Rev. Lett. 101, 240405 (2008).

[12] T. Gorin, C. Pineda, and T.H. Seligman, Phys. Rev. Lett. 99, 240405 (2007); B. Bellomo, R. Lo Franco, and G. Compagno, Phys. Rev. Lett. 99, 160502 (2007); H. Kubotani, S. Adachi, and M. Toda, Phys. Rev. Lett. 100, 240501 (2008).

[13] R. Alicki and K. Lendi, Quantum Dynamical Semigroups and Applications (Springer, New York, 1987).

[14] C.J. Myatt et al., Nature (London) 403, 269 (2000).

[15] T. Grotz, L. Heaney, and W. T. Strunz, Phys. Rev. A 74, 022102 (2006).

[16] L.J. Landau and R.F. Streater, Linear Algebr. Appl. 193, 107 (1993).

[17] F. Buscemi, G. Chiribella, and G. M. D'Ariano, Phys.
Rev. Lett. 95, 090501 (2005).

[18] T. Werlang, A. V. T Dodonov, E. I. Duzzioni, and C. J. Villas-Boas, Phys. Rev. A 78, 053805 (2008).

[19] A.R.R. Carvalho, M. Busse, O. Brodier,C. Viviescas, and A. Buchleitner, Phys. Rev. Lett. 98, 190501 (2007).

[20] R.P. Feynman and F.L. Vernon, Ann. Phys. (N.Y.) 24, 118 (1963).

[21] U. Weiss, Quantum Dissipative Systems, 3rd ed. (World Scientific Publishing, Singapore, 2008).

[22] M. Gregoratti and R. F. Werner, J. Mod. Opt. 50, 915 (2003).

[23] F. Buscemi, Phys. Lett. A 360, 256-258 (2006); K.M.R. Audenaert and S. Scheel, New J. Phys. 10, 023011 (2008); J. Watrous, Quantum Inf. Comput. 9, 406 (2009); B. Rosgen, J. Math. Phys. 49, 102107 (2008); C.B. Mendl, M.M. Wolf, Commun. Math. Phys. 289, 10571086 (2009).

[24] T.F. Havel et al., Phys. Lett. A 280, 282-288 (2001).

[25] V.I. Paulsen, Completely Bounded Maps and Operator Algebras (Cambridge University Press, Cambridge, U.K., 2002).

[26] T. Gorin, T. Prosen, T.H. Seligmann, and W.T. Strunz, Phys. Rev. A 70, 042105 (2004).

[27] See Problem 30 of R.F. Werners list of open problems in QIT, http://www.imaph.tu-braunschweig.de/qi/problems/30.html

[28] N. Johnston, D.W. Kribs, and V.I. Paulsen, Quantum Inf. Comput. 9, 16 (2009).

[29] H. Carmichael, An Open Systems Approach to Quantum Optics (Springer, New York, 1993). 